\title{
Circadian Regulation by REV-ERBa Mediates Hippocampal E-LTP in a Time-dependent Manner
}

\author{
Ja Eun Choi ${ }^{1}$, Somi Kim ${ }^{1}$, Jisu Lee ${ }^{1}$, Kyungjin Kim ${ }^{2}$ and Bong-Kiun Kaang ${ }^{1 *}$ \\ ${ }^{1}$ School of Biological Sciences, Seoul National University, Seoul 08826, \\ ${ }^{2}$ Department of Brain and Cognitive Sciences, DGIST, Daegu 42988, Korea
}

Circadian rhythms are driven by circadian oscillators, and these rhythms result in the biological phenomenon of 24-h oscillations. Previous studies suggest that learning and memory are affected by circadian rhythms. One of the genes responsible for generating the circadian rhythm is Rev-erba. The REV-ERBa protein is a nuclear receptor that acts as a transcriptional repressor, and is a core component of the circadian clock. However, the role of REV-ERBa in neurophysiological processes in the hippocampus has not been characterized yet. In this study, we examined the time-dependent role of REV-ERBa in hippocampal synaptic plasticity using Rev-erba KO mice. The KO mice lacking REV-ERBa displayed abnormal NMDAR-dependent synaptic potentiation (E-LTP) at CT12 CT14 (subjective night) when compared to their wild-type littermates. However, Rev-erba KO mice exhibited normal ELTP at CT0 CT2 (subjective day). We also found that the Rev-erba KO mice had intact late LTP (L-LTP) at both subjective day and night. Taken together, these results provide evidence that REV-ERBa is critical for hippocampal E-LTP during the dark period.

Key words: Circadian clock system, Circadian rhythm, Neuronal plasticity, Long-term potentiation

\section{INTRODUCTION}

Circadian rhythms are based on an internal timing system. The effects of circadian rhythms on learning and memory have been studied. Previous reports showed that mutations in genes responsible for generating circadian rhythms impair learning in several organisms, ranging from Drosophila [1] to mice [2,3] and humans [4]. In mammals, the suprachiasmatic nucleus (SCN) in the hypothalamus is known to be responsible for regulating circadian rhythms [5]. The RNA and protein levels of circadian oscillators are controlled by positive and negative transcriptional feedback

Received September 27, 2018, Revised October 25, 2018,

Accepted October 29, 2018

* To whom correspondence should be addressed.

TEL: 82-2-880-9024, FAX: 82-2-884-9577

e-mail:kaang@snu.ac.kr loops in the SCN [6]. The SCN, acting as a master clock, coordinates the activity of various oscillators in the brain.

REV-ERBa, which is one of the clock-modulating proteins, represses the transcription of circadian oscillators. REV-ERBa expression rhythms occur at almost $180^{\circ}$ out of phase in the SCN and the expression peaks at CT08 CT12 [6]. In addition, REV$\mathrm{ERBa}$ influences the circadian period length but is not required for circadian rhythm generation [7]. According to Gerhart-Hines et al. [8], REV-ERBa controls temperature rhythms and thermogenic plasticity. Furthermore, REV-ERBa in the ventral midbrain drives the circadian oscillations in tyrosine hydroxylase expression, thereby regulating mood [9], and is required for food entrainment [10].

Recent studies have revealed the functions of REV-ERBa in the hippocampus. The expression level of REV-ERBa in the hippocampus shows oscillation that peaks at CT08 CT12 [11], and the lack of REV-ERBa leads to alterations in hippocampus-dependent 
behaviors [12]. The regulation of the circadian clock in the hippocampus by the REV-ERBa is performed through the interaction with oligophrenin-1, which regulates dendritic spine morphology [13]. However, the role of REV-ERBa in hippocampal synaptic plasticity has not been well characterized. The present study aimed to elucidate the relationship between REV-ERBa and synaptic plasticity in the hippocampus through extracellular field recordings during subjective day and night.

\section{MATERIALS AND METHODS}

\section{Mice}

Rev-erba knock-out (KO) and wild-type (WT) mice were maintained in a $\mathrm{C} 57 \mathrm{BL} / 6 \mathrm{~J}$ background. Mice were housed at a constant room temperature with free access to food and water. The circadian time $0 \sim$ circadian time 2 (CT0 CT2) group was subjected to a normal 12-h light/12-h dark cycle, with lights switched on at 09:00. The CT12-14 group was subjected to a reversed 12-h light/12$\mathrm{h}$ dark cycle, with lights switched on at 21:00. After entrainment for $>14$ days under a reversed light-dark photoperiod, mice were maintained under the same photoperiod. All procedures were approved by the Institutional Animal Care and Use Committee of Seoul National University.

\section{Electrophysiology}

For extracellular field recordings, transverse hippocampal slices (400- $\mu$ m-thick) were prepared from brains of adult mice deeply anesthetized with isoflurane using a vibratome [14]. Hippocampal slices were incubated at $32^{\circ} \mathrm{C}$ for $30 \mathrm{~min}$ and then maintained at $28^{\circ} \mathrm{C}$ for at least $1 \mathrm{~h}$ before the experiment as described previously [15]. After recovery, the slices were placed in a recording chamber at $25^{\circ} \mathrm{C}$, and perfused with oxygenated artificial cerebrospinal fluid (ACSF) containing $124 \mathrm{mM} \mathrm{NaCl}, 2.5 \mathrm{mM} \mathrm{KCl}, 1 \mathrm{mM} \mathrm{NaH} \mathrm{PO}_{4}$,

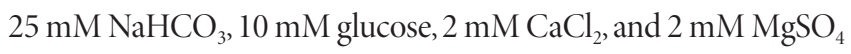
at a rate of $1 \mathrm{ml} / \mathrm{min}$. Extracellular field EPSPs (fEPSPs) were recorded from the CA1 area using a glass electrode filled with ACSF $(1 \mathrm{M} \Omega$ ). The Schaffer collateral (SC) pathway was stimulated every 30 s using concentric bipolar electrodes (MCE-100; Kopf Instruments). For measuring LTP, the stimulation intensity was adjusted to produce a fEPSP slope that was approximately $40 \%$ of the maximum slope for that slice. All LTP experiments were performed after a stable baseline was recorded. Theta burst stimulation (TBS) protocols were used to induce E-LTP and L-LTP (five pulses of $100 \mathrm{~Hz}$ repeated five times at $5 \mathrm{~Hz}$; 10 -s inter-train interval for ELTP and 10-min inter-train interval for L-LTP). Field potentials were amplified, low-pass filtered (GeneClamp 500; Axon Instruments), and then digitized (NI PCI-6221; National Instruments) for measurement. Data were monitored, analyzed online, and reanalyzed offline using the WinLTP program. Representative traces are an average of five consecutive responses and stimulus artifacts were blanked for clarity.

\section{Statistics}

Input-output curve and paired-pulse ratio data were analyzed using repeated-measures two-way ANOVA. LTP data (average of the last $5 \mathrm{~min}$ of recordings) were analyzed using an unpaired twotailed t-test. All the data are represented as mean \pm SEM.

\section{RESULTS AND DISCUSSION}

To determine whether REV-ERBa plays an important role in hippocampal synaptic plasticity, we characterized electrophysiological phenotypes in the Rev-erba KO mice. We first measured synaptic transmissions at hippocampal Schaffer-collateral-CA1-pyramidal (SC-CA1) synapses. As the REV-ERBa expression shows oscillations, the extracellular field recordings were performed during subjective day (CT0 CT2) and subjective night (CT12 CT14).

During the subjective day (CT0 CT2), the input-output relationship was similar between the Rev-erba KO mice and the WT littermates (Fig. 1A), which suggests that the lack of REV-ERBa expression does not influence basal transmission in SC-CA1 synapses. Furthermore, we performed paired-pulse ratio and confirmed that short-term plasticity is normal in Rev-erba KO mice (Fig. 1B). To understand whether REV-ERBa influences NMDARdependent synaptic plasticity, we performed early long-term potentiation (E-LTP) with theta-burst stimulation (TBS). Loss of REV-ERBa expression did not cause any impairment in the E-LTP (Fig. 1C). Together, these results suggest that the genetic deletion of REV-ERBa does not affect basal synaptic transmission, short-term plasticity, and NMDAR-dependent synaptic plasticity in the hippocampal SC-CAl pathway during the subjective day.

To further examine the association between REV-ERBa and hippocampal synaptic plasticity, we performed extracellular field recordings during the subjective night (CT12 CT14). In the Reverba KO mice, both the input-output relationship and pairedpulse ratio were normal (Fig. 2A, B), indicating that the deletion of REV-ERB $a$ does not influence basal transmission and short-term plasticity in SC-CA1 synapses. However, E-LTP appeared significantly impaired with a considerably lower potentiation magnitude (Fig. 2C). Therefore, these findings indicate that REV-ERBa has a time-dependent role in regulating the NMDAR-dependent synaptic plasticity.

The next set of experiments were performed to determine whether L-LTP varies with the time of day in the Rev-erba KO 
mice. L-LTP is a type of synaptic plasticity that is dependent on new protein synthesis and protein kinase activation [16]. Previous studies showed that the disruption of hippocampal MAPK oscillations results in theta rhythm oscillation deficits in NF1 mouse models [17]. In the Rev-erba KO mice, L-LTP was induced normally and the potentiation levels stably lasted for 3 hours. No significant differences in the magnitude of L-LTP were observed between the Rev-erba KO mice and their WT littermates, both at CT0 CT2 and at CT12 CT14 (Fig. 3A, B). These results indicate that REV-ERBa does not alter the LTP, which is dependent on protein synthesis.

The present study shows the electrophysiological role of REVERBa in the hippocampus. We found that the magnitude of E-LTP was impaired only at CT12 CT14. The subjective night-specific deficit we observed is consistent with the findings of the previous study by Schnell et al. in that the REV-ERBa expression level peaked during CT08 CT12 [11]. It is interesting that the Reverba KO mice exhibited impairments in hippocampus-dependent behaviors during CT0 CT4 [12], which includes the condition of subjective day (CT0 CT2) in this study. However, our results showed that the basic synaptic properties, short-term plasticity, and NMDAR-dependent synaptic plasticity are not altered at subjective day (CT0 CT2). The discrepancy in the behavioral and electrophysiological results can be explained by the fact that REV-ERBa is widely expressed during development; therefore, the behavioral effect observed at CT0 CT4 in the Rev-erba KO mice

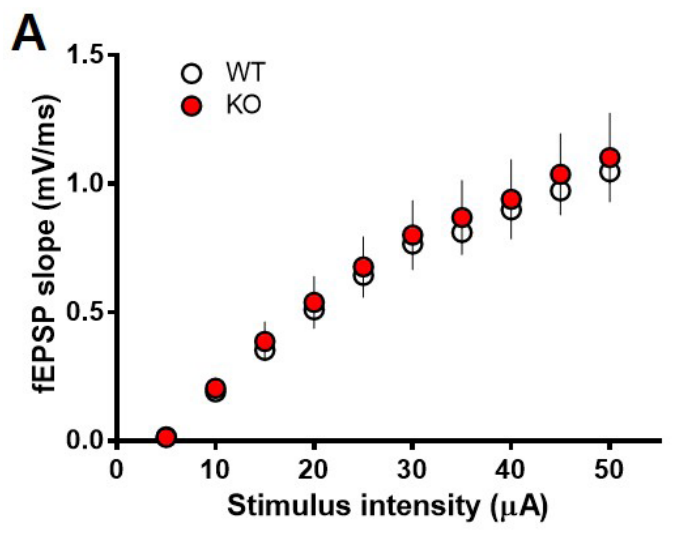

B
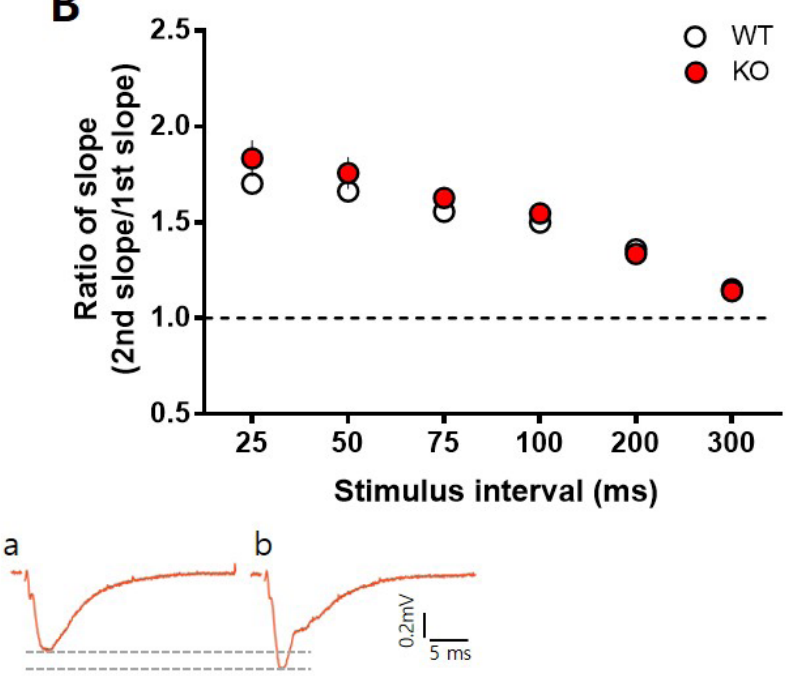

O WT

O $\mathrm{KO}$
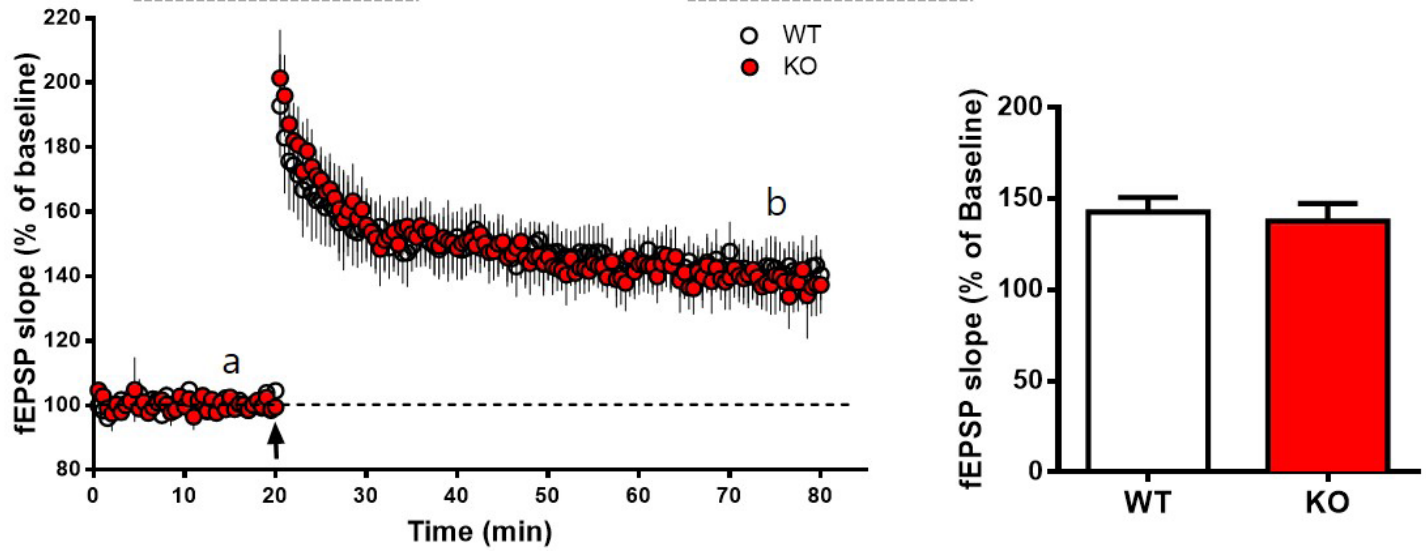

Fig. 1. Basic synaptic transmission and E-LTP are unaffected in Rev-erba KO mice on the subjective day. (A) Input-output relationships at the hippocampal Schaffer-collateral-CA1-pyramidal (SC-CA1) synapses showed no significant difference between the WT and Rev-erba KO mice (WT, 7 slices from 4 mice; Rev-erba KO, 7 slices from 4 mice; repeated-measures two-way ANOVA, effect of genotype, $F_{(1,12)}=0.7524 ; p=0.9996$ ). (B) The paired-pulse ratios showed no significant difference between the WT and KO mice. (WT, 6 slices from 4 mice; Rev-erba KO, 7 slices from 4 mice; repeated-measures two-way ANOVA, effect of genotype, $\mathrm{F}_{(1,11)}=0.4297$; $\mathrm{p}=0.1009$ ). (C) Example traces from baseline and LTP at times indicated by (a) and (b): WT (black) and KO (red). TBS-induced E-LTP was comparable between the WT and KO mice. (WT, 7 slices from 4 mice; Rev-erba KO, 6 slices from 4 mice; average fEPSP slopes for the last $5 \mathrm{~min}$; WT, $142.7 \% \pm 8.039 \%$; Rev-erba KO, $137.7 \% \pm 9.783 \%$; unpaired $t$ test, $\mathrm{p}=0.6970$ ). 
could be due to developmental brain defects. Thus, further investigation is needed to explain the association, and by performing hippocampus-dependent behavioral tasks at CT12 CT14, we may be able to explain the relationship between behavior and electrophysiology in the Rev-erba KO mice.

The hippocampus is one of the targets of dopaminergic projections from the midbrain [18]. Moreover, the CA1 region of the hippocampus expresses dopamine receptors [19], and dopamine release during specific time points is important in long-term memory consolidation [20]. A recent study by Broussard and colleagues showed that dopamine regulates synaptic plasticity in the hippocampus [21]. The Rev-erba KO mice showed dopaminergic hyperactivity $[9,12]$ and significantly higher spontaneous dopamine release from striatal tissue than the WT mice at CT12 [9].
Therefore, we might interpret that the hyperdopaminergic state of the Rev-erba KO mice may be the tentative mechanism of the significantly lower potentiation magnitude at CT12 CT14.

In several species, learning and memory, synaptic transmission, and LTP are known to show dependence on the time of day [22, 23]. According to previous studies by Jilg et al. [24] and Wang et al. [25], Per1-knockout mice showed deficits in hippocampusdependent long-term spatial learning and Per2-mutant mice exhibited impairments in the hippocampal LTP. Our data reveal that another circadian oscillator, REV-ERBa, is involved in phasedependent hippocampal LTP dynamics. In addition, the Rev-erba KO mice exhibited impaired E-LTP at CT12 CT14 with intact L-LTP at both time points, which requires new protein synthesis. The result can be explained by the study by Sakai and colleagues,
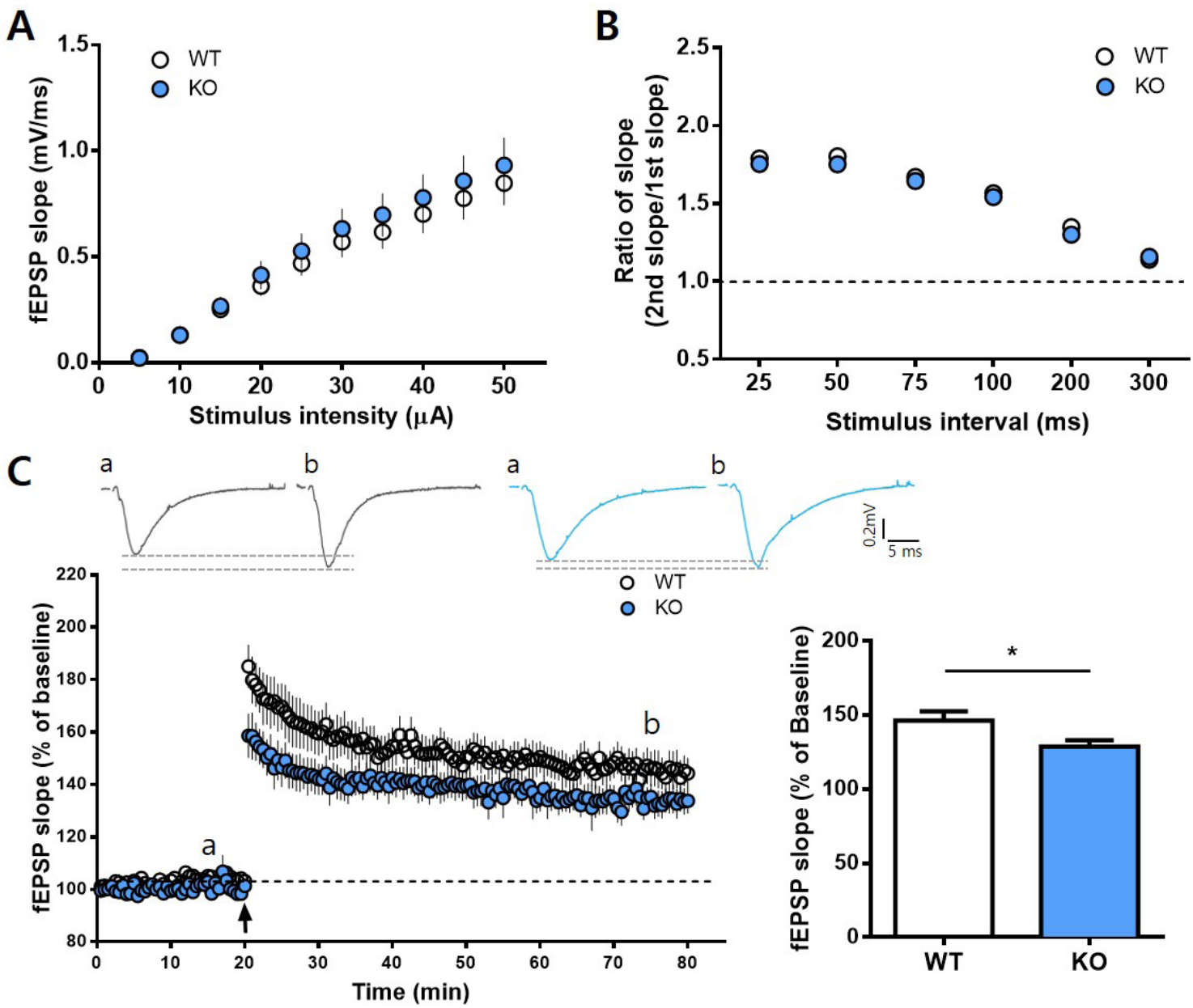

b
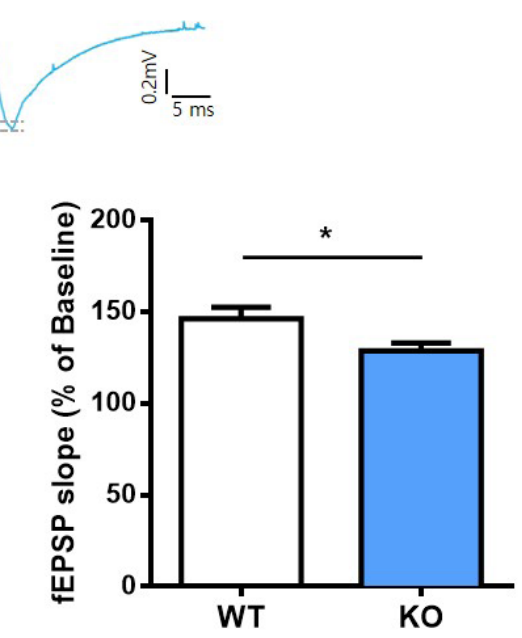

Fig. 2. E-LTP is impaired in Rev-erba KO mice during the subjective night. (A) The input-output relationships at SC-CA1 synapses showed no significant differences between WT and Rev-erba KO mice at CT12 13. (WT, 12 slices from 7 mice; Rev-erba KO, 12 slices from 7 mice; repeated-measures two-way ANOVA, effect of genotype, $\mathrm{F}_{(1,22)}=0.5963 ; \mathrm{p}=0.9631$ ). (B) Paired-pulse ratios showed no significant differences between the WT and $\mathrm{KO}$ mice at CT12 13. (WT, 12 slices from 7 mice; Rev-erba KO, 13 slices from 7 mice; repeated-measures two-way ANOVA, effect of genotype, $\mathrm{F}_{(1,22)}=0.6569$; $\mathrm{p}=0.7418$ ). (C) Representative traces from baseline and LTP at times indicated by (a) and (b): WT (black) and KO (blue). TBS-induced E-LTP was significantly lower in the Rev-erba KO mice (WT, 11 slices from 7 mice; Rev-erba KO, 11 slices from 7 mice; average fEPSP slopes for the last 5 min; WT, $146.4 \% \pm 6.073 \%$; Rev-erba KO, $128.7 \% \pm 4.288 \%$; unpaired $t$-test, $\mathrm{p}=0.0274) .{ }^{*} \mathrm{p}<0.05$. 

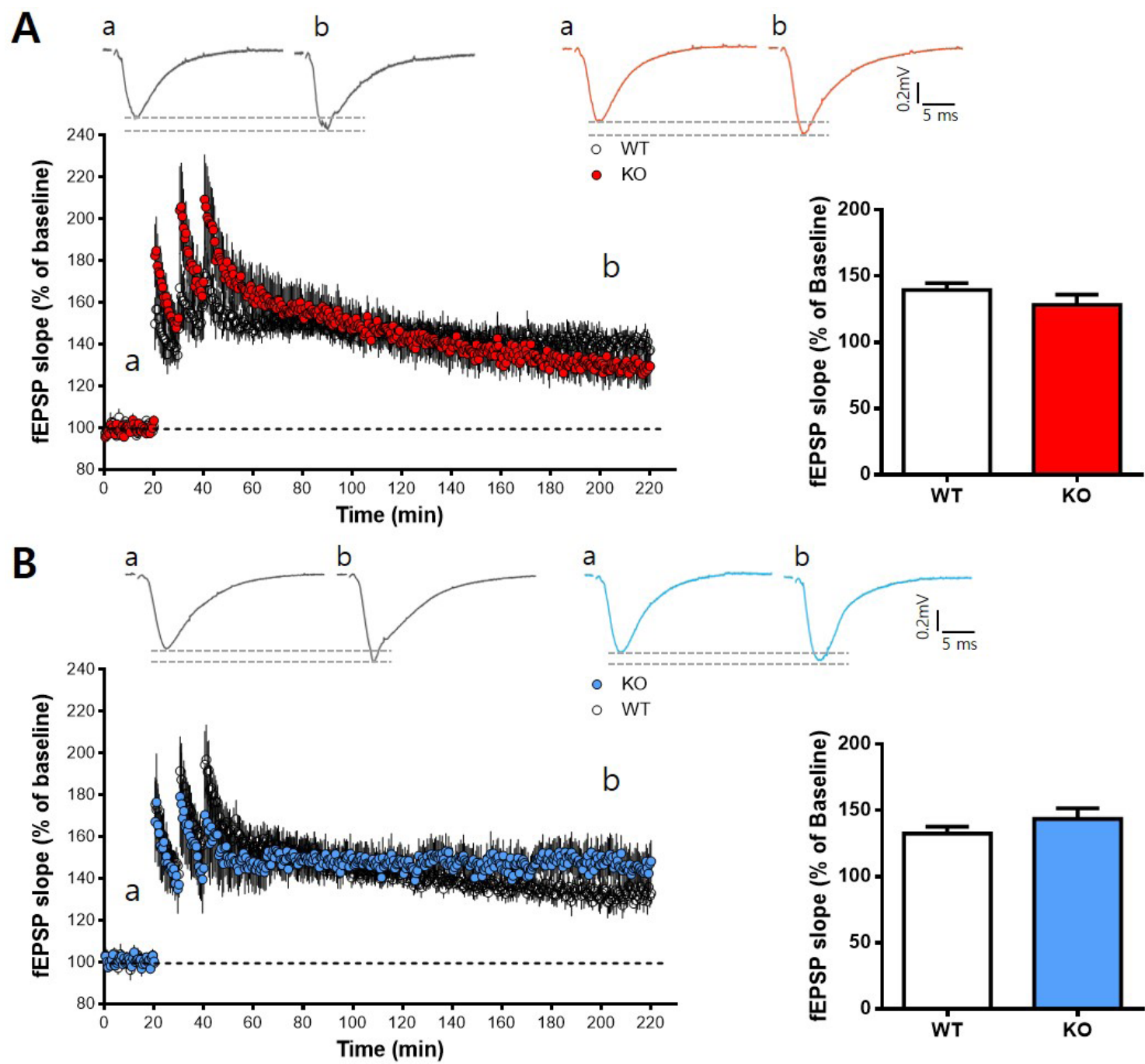

b
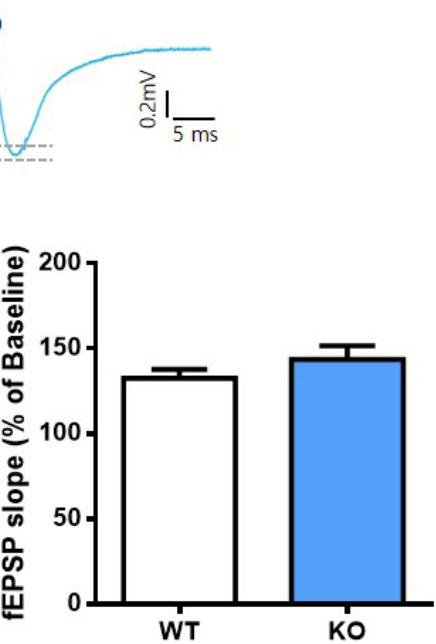

Fig. 3. L-LTP is unaffected in the Rev-erba KO mice during the subjective day and night. Example traces from baseline and LTP at times indicated by (a) and (b): WT (black) and KO (red or blue). (A) During the subjective day, Rev-erba KO mice did not exhibit any impairment in the TBS-induced hippocampal L-LTP, and the potentiation level was maintained at a level comparable with that in the WT mice for $3 \mathrm{~h}$ (WT, 7 slices from 6 mice; Rev-erba KO, 6 slices from 6 mice; average fEPSP slopes for the last 5 min; WT, 139.6\% $\pm 5.199 \%$; Rev-erba KO, 128.2\% $\pm 7.817 \%$; unpaired $t$ test; p=0.2388). (B) During the subjective night, Rev-erba KO mice did not show any deficits in the TBS-induced hippocampal L-LTP (WT, 6 slices from 6 mice; Rev-erba KO, 6 slices from 6 mice; average fEPSP slopes for the last $5 \mathrm{~min}$; WT, 132.6\% $\pm 5.213 \%$; Rev-erba KO, 143.6\% $\pm 7.963 \%$; unpaired $t$ test; $\mathrm{p}=0.2736$ ).

in which they showed that long-term memory is independent of the circadian rhythm [1]. However, the exact mechanism underlying the regulation of protein synthesis-dependent and protein synthesis-independent synaptic plasticity by Rev-erba is yet to be discovered.

In summary, REV-ERBa is an important circadian protein for regulating the magnitude of hippocampal E-LTP during the subjective night.

\section{ACKNOWLEDGEMENTS}

This work was supported by the National Honor Scientist Pro- gram (NRF-2012R1A3A1050385) of Korea. J.E.C was supported by a Fellowship for Fundamental Academic Fields by Seoul National University.

\section{REFERENCES}

1. Sakai T, Tamura T, Kitamoto T, Kidokoro Y (2004) A clock gene, period, plays a key role in long-term memory formation in Drosophila. Proc Natl Acad Sci U S A 101:16058-16063.

2. Garcia JA, Zhang D, Estill SJ, Michnoff C, Rutter J, Reick M, Scott K, Diaz-Arrastia R, McKnight SL (2000) Impaired cued and contextual memory in NPAS2-deficient mice. Science 
288:2226-2230.

3. Eckel-Mahan KL (2012) Circadian oscillations within the hippocampus support memory formation and persistence. Front Mol Neurosci 5:46.

4. Wright KP Jr, Hull JT, Czeisler CA (2002) Relationship between alertness, performance, and body temperature in humans. Am J Physiol Regul Integr Comp Physiol 283:R1370R1377.

5. Takahashi JS, Hong HK, Ko CH, McDearmon EL (2008) The genetics of mammalian circadian order and disorder: implications for physiology and disease. Nat Rev Genet 9:764-775.

6. Reppert SM, Weaver DR (2002) Coordination of circadian timing in mammals. Nature 418:935-941.

7. Preitner N, Damiola F, Lopez-Molina L, Zakany J, Duboule D, Albrecht U, Schibler U (2002) The orphan nuclear receptor Rev-erba controls circadian transcription within the positive limb of the mammalian circadian oscillator. Cell 110:251260.

8. Gerhart-Hines Z, Feng D, Emmett MJ, Everett LJ, Loro E, Briggs ER, Bugge A, Hou C, Ferrara C, Seale P, Pryma DA, Khurana TS, Lazar MA (2013) The nuclear receptor Rev-erba controls circadian thermogenic plasticity. Nature 503:410413.

9. Chung S, Lee EJ, Yun S, Choe HK, Park SB, Son HJ, Kim KS, Dluzen DE, Lee I, Hwang O, Son GH, Kim K (2014) Impact of circadian nuclear receptor Rev-erba on midbrain dopamine production and mood regulation. Cell 157:858-868.

10. Delezie J, Dumont S, Sandu C, Reibel S, Pevet P, Challet E (2016) Rev-erba in the brain is essential for circadian food entrainment. Sci Rep 6:29386.

11. Schnell A, Chappuis S, Schmutz I, Brai E, Ripperger JA, Schaad O, Welzl H, Descombes P, Alberi L, Albrecht U (2014) The nuclear receptor Rev-erba regulates Fabp7 and modulates adult hippocampal neurogenesis. PLoS One 9:e99883.

12. Jager J, O’Brien WT, Manlove J, Krizman EN, Fang B, GerhartHines Z, Robinson MB, Klein PS, Lazar MA (2014) Behavioral changes and dopaminergic dysregulation in mice lacking the nuclear receptor Rev-erba. Mol Endocrinol 28:490-498.

13. Valnegri P, Khelfaoui M, Dorseuil O, Bassani S, Lagneaux C, Gianfelice A, Benfante R, Chelly J, Billuart P, Sala C, Passafaro M (2011) A circadian clock in hippocampus is regulated by interaction between oligophrenin-1 and Rev-erba. Nat Neurosci 14:1293-1301.

14. Lim CS, Nam HJ, Lee J, Kim D, Choi JE, Kang SJ, Kim S, Kim H, Kwak C, Shim KW, Kim S, Ko HG, Lee RU, Jang EH, Yoo J,
Shim J, Islam MA, Lee YS, Lee JH, Baek SH, Kaang BK (2017) PKCa-mediated phosphorylation of LSD1 is required for presynaptic plasticity and hippocampal learning and memory. Sci Rep 7:4912.

15. Kim S, Yu NK, Shim KW, Kim JI, Kim H, Han DH, Choi JE, Lee SW, Choi DI, Kim MW, Lee DS, Lee K, Galjart N, Lee YS, Lee JH Kaang BK (2018) Remote Memory and Cortical Synaptic Plasticity Require Neuronal CCCTC-Binding Factor (CTCF). J Neurosci 38:5042-5052.

16. Kandel ER, Dudai Y, Mayford MR (2014) The molecular and systems biology of memory. Cell 157:163-186.

17. Chen L, Serdyuk T, Yang B, Wang S, Chen S, Chu X, Zhang X, Song J, Bao H, Zhou C, Wang X, Dong S, Song L, Chen F, He G, He L, Zhou Y, Li W (2017) Abnormal circadian oscillation of hippocampal MAPK activity and power spectrums in NF1 mutant mice. Mol Brain 10:29.

18. Gasbarri A, Packard MG, Campana E, Pacitti C (1994) Anterograde and retrograde tracing of projections from the ventral tegmental area to the hippocampal formation in the rat. Brain Res Bull 33:445-452.

19. Mu Y, Zhao C, Gage FH (2011) Dopaminergic modulation of cortical inputs during maturation of adult-born dentate granule cells. J Neurosci 31:4113-4123.

20. Rossato JI, Bevilaqua LR, Izquierdo I, Medina JH, Cammarota M (2009) Dopamine controls persistence of long-term memory storage. Science 325:1017-1020.

21. Broussard JI, Yang K, Levine AT, Tsetsenis T, Jenson D, Cao F, Garcia I, Arenkiel BR, Zhou FM, De Biasi M, Dani JA (2016) Dopamine regulates aversive contextual learning and associated in vivo synaptic plasticity in the hippocampus. Cell Reports 14:1930-1939.

22. Lyons LC, Rawashdeh O, Eskin A (2006) Non-ocular circadian oscillators and photoreceptors modulate long term memory formation in Aplysia. J Biol Rhythms 21:245-255.

23. Chaudhury D, Wang LM, Colwell CS (2005) Circadian regulation of hippocampal long-term potentiation. J Biol Rhythms 20:225-236.

24. Jilg A, Lesny S, Peruzki N, Schwegler H, Selbach O, Dehghani F, Stehle JH (2010) Temporal dynamics of mouse hippocampal clock gene expression support memory processing. Hippocampus 20:377-388.

25. Wang LM, Dragich JM, Kudo T, Odom IH, Welsh DK, O'Dell TJ, Colwell CS (2009) Expression of the circadian clock gene Period2 in the hippocampus: possible implications for synaptic plasticity and learned behaviour. ASN Neuro 1:e00012. 